\title{
Complejidad territorial en caletas de pescadores artesanales de Chiloé (Chile): aportes para el manejo costero
}

\section{Territorial complexity in fishermen's coves of Chiloé (Chile): contributions for coastal management}

\author{
Francisco Ther-Ríos ${ }^{1}$ https://orcid.org/0000-0002-6043-4340 \\ Paula Salinas-Vilches ${ }^{2}$ https://orcid.org/0000-0003-3670-8214 \\ Gonzalo Gajardo-Gálvez ${ }^{3}$ https://orcid.org/0000-0001-8634-141X \\ Zamir Bugueño ${ }^{4}$ https://orcid.org/0000-0002-1900-3215 \\ Claudio Gajardo-Cortés ${ }^{5}$ https://orcid.org/0000-0001-6155-5171 \\ Mauricio Ceballos-Cardona ${ }^{6}$ https://orcid.org/0000-0002-2416-2907 \\ Jaime Valderrama-Bravo ${ }^{7}$ https://orcid.org/0000-0002-1813-0183 \\ Jaime A. Cursach ${ }^{8}$ https://orcid.org/0000-0002-3251-4474 \\ Carlos Hidalgo-Garrido9 https://orcid.org/0000-0002-3358-5864 \\ ${ }^{1}$ Departamento de Arquitectura, Universidad de Los Lagos, Casilla 933 - Osorno, CHILE. \\ Email: fther@ulagos.cl \\ ${ }^{2}$ Consultora Independiente. CHILE. Email: geo.psalinas@gmail.com \\ ${ }^{3}$ Laboratorio de Genética y Acuicultura, Departamento de Ciencias Biológicas y Biodiversidad, Universidad de Los Lagos. \\ Email: ggajardo@ulagos.cl \\ ${ }^{4}$ Departamento de Ciencias Sociales, Universidad de Los Lagos. CHILE. Email: zamir.bugueno@ulagos.cl \\ ${ }^{5}$ Estudiante Doctorado en Ciencias Sociales, Universidad de Los Lagos. CHILE. Email: clgajardocortes@gmail.com \\ ${ }^{6}$ Consultor independiente. CHILE. Email: m.ceballos.cardona@gmail.com \\ ${ }^{7}$ Ministerio de Defensa. CHILE. Email: valderrama.jaime@gmail.com \\ ${ }^{8}$ Consultor Independiente. CHILE. Email: jcurval@gmail.com \\ ${ }^{9}$ Estudiante Doctorado en Ciencias de la Complejidad Social, Universidad del Desarrollo. CHILE. \\ Email:chidalgarrido@gmail.com
}

\section{Resumen}

Las comunidades costeras de pesca artesanal (conocidas a pequeña escala como "caletas") son sistemas socioecológicos complejos que se adaptan a las perturbaciones dependiendo de su complejidad y propiedades inherentes. La capacidad de evaluar y monitorear esta respuesta adaptativa proporciona información sobre la sostenibilidad de las caletas, necesaria para una planificación estratégica y territorial. Seis caletas ubicadas en el archipiélago de Chiloé, sur de Chile, sirvieron de modelo para identificar la complejidad territorial. En este artículo se identificaron 4 criterios y 16 subcriterios (utilizando el software Expert Choice) como descriptores adecuados de su complejidad y se clasificaron jerárquicamente según su importancia (\%). Todas estas variables se fusionaron en un Índice de Complejidad Territorial (ICT), un estimador cualicuantitativo de la sostenibilidad de las caletas. Un Análisis de Componentes Principales incluyó todos los subcriterios y caracterizó la complejidad de las caletas. Este artículo demuestra que la complejidad requiere un análisis integrado de las variables relevantes para el monitoreo de las trayectorias evolutivas de las caletas, ante lo cual, el ICT destaca como una herramienta cuantitativa conveniente para la planificación estratégica y territorial.

Palabras claves: Índice de Complejidad Territorial, pesca artesanal, resiliencia, sistemas socioecológicos, sustentabilidad.

\begin{abstract}
Coastal artisanal fishing communities (small scale coves or "caletas") are complex socio-ecological systems that adapt to perturbations depending on their inherent complexity and properties. The ability to evaluate and monitor such adaptive response provides information on caletas sustainability, required for reliable territorial and strategic planning. Six caletas from different geographic locations in Chiloe Island, southern Chile, served as a model to identify territorial complexity. This article identified 4 criteria and 16 sub-criteria (using Expert Choice software) as suitable descriptors of their complexity, and hierarchically ranked them according to importance (\%). All these variables were merged into a Territorial Complexity Index (ICT), a quali-quantitative estimator of caletas sustainability. A principal Component Analysis, including all sub-criteria, further characterized caletas complexity. This article showed that complexity needs an integrated analysis of the relevant variables to monitor evolutionary trajectories of caletas, and thus ICT is highlighted as a convenient quantitative tool for territorial and strategic planning.
\end{abstract}

Keywords: Territorial Complexity Index, artisanal fisheries, resilience, social-ecological system, sustainability.

Recibido: 15 julio 2017. Aceptado: 24 julio 2018 


\section{Introducción}

Las transformaciones sociales, ambientales y científicas de las últimas décadas han modificado sustancialmente los escenarios de las sociedades costeras, de la actividad pesquera y, particularmente, de la pesca artesanal. Se trata, en términos generales, de un escenario desalentador (Pauly, 2005; Kelleher, 2005; Worm et al., 2006), en el que pareciera que las sociedades costeras de pequeña escala aún no reciben un tratamiento acorde a los beneficios económicos, alimenticios y el escaso daño medioambiental que representa (FAO, 2000; Zeller et al., 2005; Béné et al., 2007). La aceleración de estas transformaciones ha derivado en el aumento de la vulnerabilidad de muchas de estas sociedades costeras, planteando urgentes desafíos a los planteamientos teórico-metodológicos de la ciencia y la gestión costera. En este sentido, avances en el modelamiento de la diversidad y la planificación estratégica del desarrollo territorial en sociedades litorales han asumido parte de las problemáticas denunciadas por los cuestionamientos respecto de las políticas de manejo y desarrollo social convencionales (Holling, 1973; Gunderson et al., 1995; Alcalá, 1999; García-Allut, 1999; Ostrom, 2000; Berkes, 2008). Se ha indicado que resulta fundamental traspasar los avances de diversas ciencias respecto de conceptos claves (sustentabilidad, sistemas socioecológicos, resiliencia y conocimiento ecológico local, entre otros) hacia organismos de planificación, ordenamiento y gestión. Esto, además, adquiere mayor urgencia si consideramos que el capitalismo de recursos, el centralismo y la visión sectorial prevalecen y mantienen una desconexión entre las prácticas para el desarrollo y las dinámicas de uso y apropiación de recursos que existen efectivamente en los territorios costeros. Un importante desafío consiste en construir herramientas apropiadas para avanzar desde la base de las dinámicas socioecológicas hacia la superficie de las planificaciones, procurando convertirlas en planes que constituyan modelaciones representativas de la diversidad biocultural litoral.

Con miras a contribuir al diseño y construcción de herramientas de modelamiento propicias para dar cuenta de las intrincadas relaciones socioambientales que existen en la gestión de la sustentabilidad, en este artículo nos hemos propuesto el abordaje de dos problemáticas particulares: 1) el desencuentro entre estrategias y discursos presentes en las dinámicas de interacción territorial, producto de la confluencia en zonas costeras de actividades de pesca artesanal, el incipiente desarrollo turístico, la industria de productos marinos y la introducción de ciencia y tecnología, en el marco de un creciente capitalismo de recursos en el país, articulados y desarticulados en diversas redes de actores y relaciones (Barragán et al., 2005; Marín y Berkes, 2010); y 2) la inminente llegada al litoral chileno de la crisis mundial de recursos costeros (Pauly, 2005; Pauly et al., 2006; Jentoft y Eide, 2011).

Los territorios costeros del sur de Chile presentan una elevada biodiversidad, donde actualmente coexisten prácticas tradicionales de comercio y extracción, junto a pujantes procesos modernizadores que han evidenciado fallas en los modelos de gestión medioambiental, decantando incluso en severas crisis económico-ecológicas (Montero, 2004; Barton y Floysand, 2010; Gajardo y Ther, 2011; Bustos, 2013). En este escenario, resulta de primordial importancia interrogarse sobre la existencia de diversas capacidades y estrategias de resiliencia y sustentabilidad de las comunidades costeras, y si es posible imaginar una metodología apropiada que dé cuenta de la complejidad de esas capacidades y vínculos socioecológicos. Por ello, el objetivo de este artículo es mostrar una herramienta de evaluación de la relación entre biodiversidad y sociodiversidad de los territorios costeros, a la que hemos denominado Índice de Complejidad Territorial (ICT). El ICT fue aplicado en seis caletas de pesca artesanal del sur de Chile, consideradas como sistemas socioecológicos que reflejan las interrelaciones y adaptaciones entre los sistemas biofísico y social (Berkes y Folke, 1998; Kallis y Norgaard, 2010). Evaluando la hipótesis de que el ICT permitirá evidenciar la existencia y las diferencias de la diversidad intra e inter caletas artesanales, junto con ser una buena herramienta para evaluar las diversas estrategias y capacidad de respuesta o resiliencia de estos territorios, ante variables socioculturales, económicas y ambientales a las que se ven expuestas (Pauly, 2006; Perry et al., 2010; Hall, 2011; Defeo et al., 2013). A lo largo del texto se detalla la metodología de su diseño y se explorán los alcances de este instrumento. 


\section{Conceptualización del ICT}

\section{Sistemas socioecológicos}

Durante las últimas décadas del siglo $\mathrm{XX}$, los esquemas científicos y de gestión de recursos naturales se enfrentaron a críticas desde diversos frentes (Gunderson et al., 1995; National Research Council, 2002). La ciencia convencional se sostuvo en el análisis de las relaciones socioambientales a partir de la yuxtaposición de sus componentes, es decir, el sistema social y el sistema natural. En respuesta a esto ha aparecido un enfoque que aboga por redefinir esta relación, relevando el carácter interdependiente (Berkes y Folke, 1998; Gunderson y Holling, 2002) e incluso coevolutivo de los sistemas sociales y naturales (Van der Leeuw, 2000; Plummer, 2009; Kallis y Norgaard, 2010). Aparece así el concepto de sistema socioecológico (SES), para el que Berkes et al. (2003) ofrecen una serie de características por corresponder a un sistema complejo: no-linealidad, emergencia, incertidumbre, escala y autoorganización. Berkes y Folke (1998) han propuesto un modelo heurístico que permite comprender de mejor manera la constitución y dinámica de un SES, el que será de vital importancia puesto que posteriormente en torno a él se diseña la herramienta metodológica ICT. El modelo de los autores comprende cuatro elementos para la descripción de características y relaciones en un SES: i) ecosistemas: particularmente la descripción de la estructura o dinámica de los componentes físicos y biológicos; ii) conocimiento local: o el conocimiento de su medio ambiente y recursos que cada usuario adquiere y que, en caso de tratarse de un producto de acumulación histórica, puede referirse como conocimiento local tradicional (LEK); iii) personas y tecnología: descripción del sistema social y la tecnología que emplea en las relaciones socioecológicas; iv) instituciones de derechos de propiedad: descripción de las instituciones de diversas escalas que organizan las relaciones socioecológicas.

\section{Sustentabilidad y resiliencia}

El concepto desarrollo sustentable irrumpe en el debate científico y político internacional tras su inclusión dentro del Informe Brundtland, publicado el año 1987 (WCED, 1987). Si bien, originalmente habría sido definido como el desarrollo que "...Meet the needs of the present generation without compromising the ability of future generations to meet their own needs" (WCED, 1987), la efervescencia política que desató provocó que se transformase en objeto de intenso debate científico, diversificando las posturas respecto de sus características y ampliando la gama de definiciones. El concepto de sustentabilidad es el resultado de un proceso de reflexión y toma de conciencia respecto de los riesgos ecológicos asociados a los procesos de globalización, y de la profunda interrelación entre los problemas y crisis ambientales y las políticas de desarrollo. Pese a la polisemia que ha adquirido, Haughton (1999) sintetiza sus elementos principales en cinco ideas relacionadas con la equidad: "futurity - inter-generational equity; social justice - intra-generational equity; transfrontier responsibility - geographical equity; procedural equity - people treated openly and fairly; interspecies equity - importance of biodiversity". El concepto de equidad y, en términos más generales, sustentabilidad, resultan fundamentales en el debate actual por constituir una clave y una guía para enfrentar las crisis ecológicas de diversas escalas, y en el que confluyen las dimensiones ecológicas, sociales y económicas. En esta investigación entenderemos la sustentabilidad como "...mantaining the capacity of ecological systems to support social and economic systems" (Berkes et al., 2003). Uno de los mayores desafíos que implica es el diseño de herramientas que den cuenta de distintos tipos y grados de sustentabilidad, que permitan colaborar en las instancias de mejora de su gestión, a través de la focalización de sus iniciativas y esfuerzos. En este sentido, en nuestra investigación el concepto de sustentabilidad adquiere importancia puesto que corresponde a una referencia para los sistemas socioecológicos que aquí serán analizados como casos de estudio, y de sus capacidades de respuesta ante los desafíos de las crisis medioambientales.

La resiliencia es un concepto clave que permitirá enlazar de mejor manera el de SES y sustentabilidad, y que también se encuentra reflejado en el modelo metodológico del ICT. La perspectiva de la resiliencia aparece cuando Holling (1973) demuestra la existencia de múltiples dominios de estabilidad o equilibro. Desde aquí, la resiliencia fue presentada como la capacidad de un sistema para persistir en 
un dominio de equilibrio/equilibrado, dentro de un contexto de cambio (Folke, 2006). Rápidamente, este concepto desborda los límites de la biología y comienza a ser utilizado en antropología (Vayda y McCay, 1975), psicología ambiental (Lamson, 1986), modelación de sistemas complejos de interacción entre humanos y medio ambiente (Constanza et al. 1993), geografía humana (Zimmerer, 1994), entre otros. Con ello, el concepto de resiliencia se refiere a la capacidad de respuesta y manejo de determinados sistemas ante transformaciones o cambios (Folke, 2006; Zoly y Healy, 2012), influidos tanto por forzantes externas como internas (González et al., 2008). En relación a los SES, Young et al. (2006) han enfatizado el rol de la resiliencia como marco para el análisis de perturbaciones de diversas escalas, por ejemplo, las transformaciones y crisis económicas y ecológicas globales. De esta manera, a mayor resiliencia de un SES, mayor capacidad para absorber perturbaciones, adaptarse a los cambios y comportarse como un sistema sustentable (manteniendo tasas de equilibrio), mientras que a menor resiliencia habrá mayor vulnerabilidad a las perturbaciones (Berkes et al., 2003; Olsson, 2003). Como se verá más adelante, en este artículo procuramos que la herramienta del ICT se encuentre vinculada a estos conceptos de SES, sustentabilidad y resiliencia. El objetivo es que mediante el ICT se obtenga un indicador que dé cuenta de la complejidad en las relaciones socioecológicas y las capacidades de resiliencia de los SES de caletas de pescadores artesanales, permitiendo evaluar y comparar sus cualidades sustentables.

\section{Complejidades territoriales y planificación}

La complejidad territorial, como concepto, ha sido poco utilizada en el quehacer científico. Pese a esto, existen trabajos que se relacionan de manera más o menos directa con la temática (Giraut, 2011). Papadimitriou (2012a, b) utiliza el concepto Complejidad asociado al de Paisaje, entendido como la configuración estructural o espacial de un lugar. Dicho autor modeliza la complejidad del paisaje como herramienta para el manejo del uso del suelo, en una perspectiva directamente ligada al ordenamiento territorial. En este sentido, la modelización de este atributo se presenta como un medio para avanzar en la política pública en torno al ordenamiento y gestión medioambiental de sistemas urbanos. El modelo de Papadimitriou (2012a,b) esquiva el tratamiento y modelización de las características cualitativas y socioculturales que componen la complejidad de los paisajes. Mientras que Laterra et al. (2012) utilizan el concepto de complejidad espacial asociado a sectores rurales, considerando que la complementación entre distintos tipos de servicios ecosistémicos y la fuerza de sus relaciones disminuye debido a la mayor o menor complejidad de los paisajes rurales. De esta manera, la complejidad es asumida como facilitadora o restrictiva de los intercambios entre los servicios ecosistémicos. También es posible identificar variantes en la construcción de metodologías de planificación y ordenamiento territorial; así, algunas se materializan desde el flujo bottom-up o top-down (Gunderson y Holling, 2002), mientras que otras se centran en la caracterización de modos de vida sustentable (Cormont et al., 2016), calificación jerárquica (Gristo et al., 2015), teoría de orientación (Bossel, 2001), teoría presión-estado-respuesta (Liu y Hao, 2017), evaluación del bienestar (PrescottAllen, 2001), manejo territorial y evaluación de sustentabilidad (Reed et al., 2015).

Los actuales procesos de transición, transformación y cambio social en la esfera de lo local están significando una enorme complejidad para su análisis, en que el territorio representa el tiempo espacializado de las actividades humanas cuya expresión está dada por imbricaciones contextuadas, donde el análisis del territorio y sus transformaciones exige un fuerte reconocimiento de lo local y su interacción con lo global (Ther-Ríos, 2006). Así, para comprender o interpretar mejor los territorios, se deben tomar en cuenta las múltiples dimensiones sociales que emergen en la complejidad misma en la cual se desenvuelven las personas con sus instituciones, en su vínculo con sus límites materiales "naturales" o "creados" (Kollmannm, 2005, Urquieta, et al., 2017). Por lo cual, y desde una perspectiva socioecológica (Fraser et al., 2006; Graymore et al., 2008), nuestra propuesta busca revalorizar los conocimientos locales y posibilitar la aplicación de mayores variables en el desarrollo de modelos de sustentabilidad en caletas de pescadores artesanales. 


\section{Metodología para el diseño y construcción del ICT}

La estrategia metodológica utilizada en esta investigación contempló una etapa inicial de levantamiento de información (primaria/secundaria) y el posterior diseño y construcción del ICT.

\section{Descripción de las caletas de pescadores artesanales estudiadas}

Dentro de la Patagonia chilena se ubica la ecorregión Chiloense (41-47o $\mathrm{S}$ ), en cuya porción norte se encuentra el archipiélago de Chiloé (42-43 $\mathrm{S}$; 73-75० O), compuesto por la Isla Grande de Chiloé y más de 40 islas menores, en un clima muy lluvioso (4000 a $7000 \mathrm{~mm}$ por ańo) con influencia oceánica (Hucke-Gaete et al., 2010). Su geografía es dominada por montańas de baja altitud que conforman las últimas elevaciones importantes de la cordillera de la Costa, en cuyo litoral oriental existen fiordos, canales, islas y bahías muy cerradas (p.e., mar interior), mientras que su borde poniente es una costa expuesta al océano Pacífico (p.e., mar abierto) (Figura 1).
El mar interior de Chiloé es un territorio de mar y bordemar cargado de puertos y lugares de llegada, en el cual confluyen distintos trayectos marítimos entre islas y sectores poblados que estructuran trayectorias y dinámicas plasmadas en memorias del pasado e imaginarios del futuro (Ther-Ríos, 2011). Por otra parte, existen diferencias ambientales considerables entre los ambientes de mar interior y mar abierto de esta zona, como son la salinidad del agua (por descargas de ríos desde la cordillera de los Andes) y la altura y amplitud del régimen de mareas (Davies, 1972; Lara et al., 2010).

Se trabajó con seis caletas de pescadores artesanales de Chiloé, ubicadas tanto en la costa de mar abierto como en el mar interior (Figura 1). Las caletas de

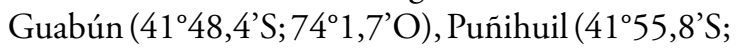
$\left.74^{\circ} 1,5^{\prime} \mathrm{O}\right)$ y Cucao $\left(42^{\circ} 38,1^{\prime} \mathrm{S} ; 7^{\circ} 6,8^{\prime} \mathrm{O}\right)$ se ubican en la costa de mar abierto (ver Figura 1). Mientras que las caletas de Tenaún ( $\left.42^{\circ} 19,8^{\prime} S ; 73^{\circ} 22,1^{\prime} \mathrm{O}\right)$, El Manzano $\left(42^{\circ} 0,6^{\prime} S ; 72^{\circ} 38,4^{\prime} \mathrm{O}\right)$ y Puntilla $\mathrm{Pi}$ chicolo $\left(42^{\circ} 0,2^{\prime} S ; 72^{\circ} 35,6^{\prime} \mathrm{O}\right)$ se ubican en el mar interior (ver Figura 1). En la Tabla 1 se entrega una caracterización sistematizada que describe estas seis caletas.

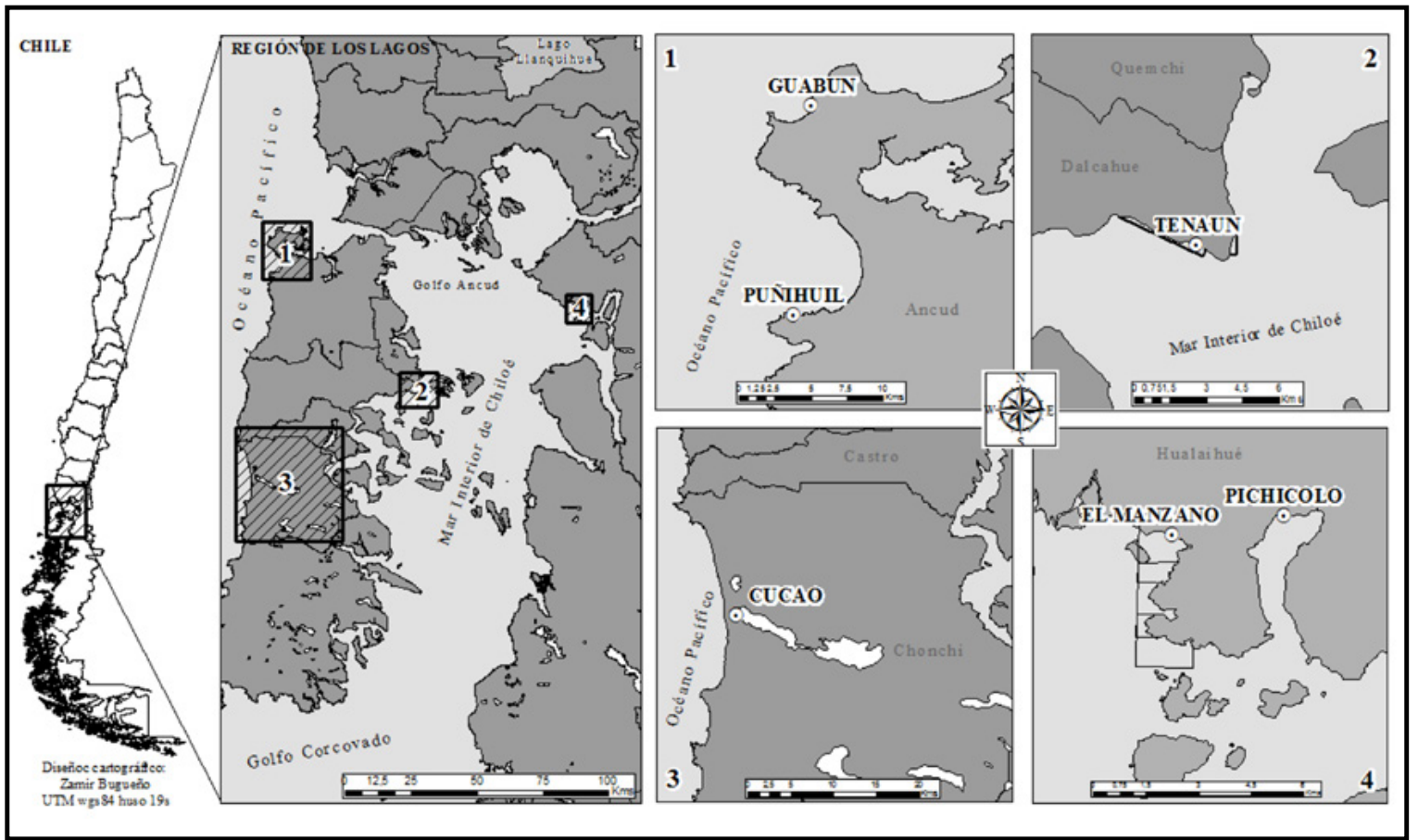

Figura 1. Ubicación geográfica de las seis caletas de pescadores artesanales consideradas para la construcción del ICT. 
Tabla 1. Caracterización de las caletas de pesca artesanal estudiadas, indicando su número de habitantes.

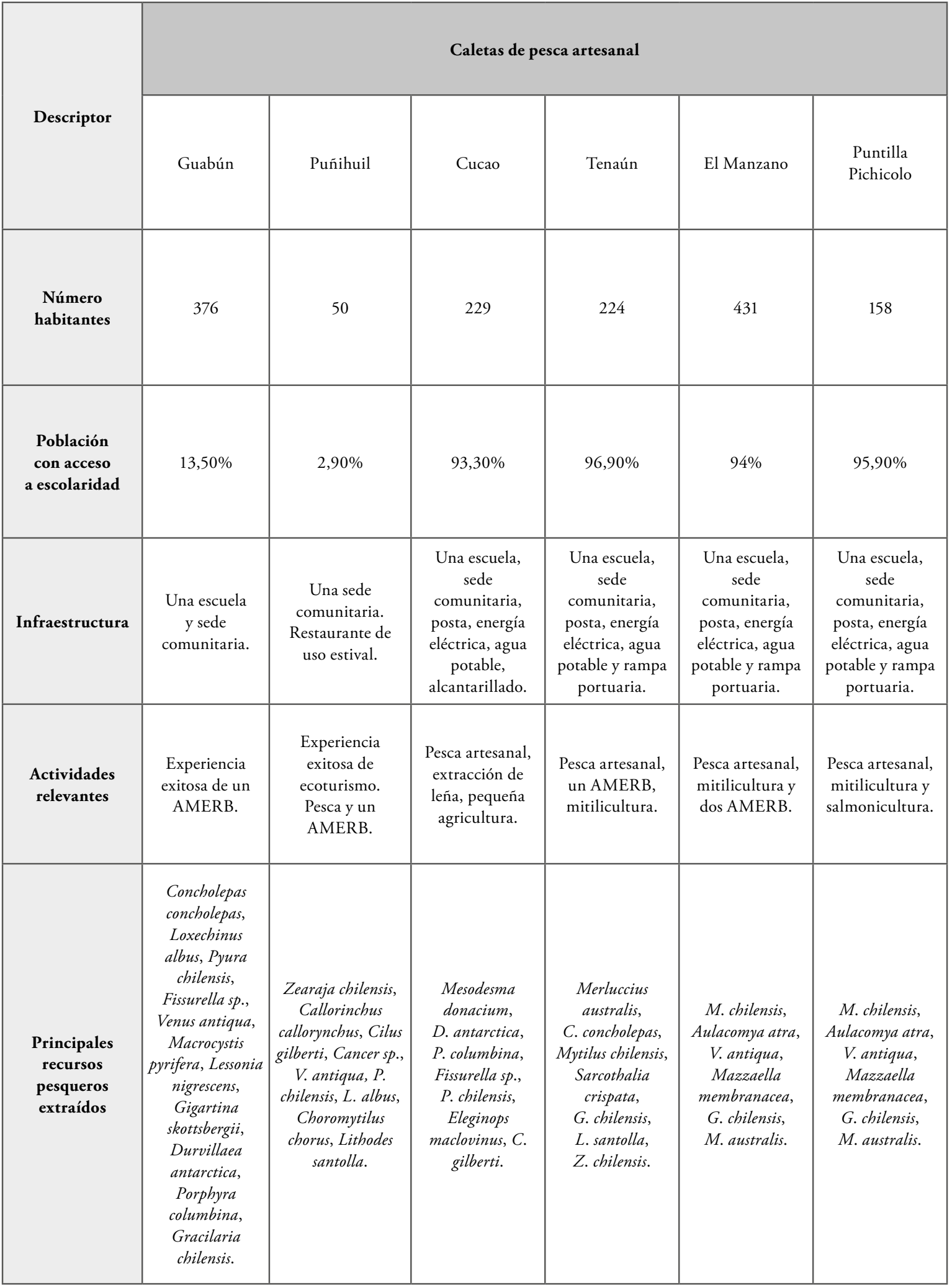




\begin{tabular}{|c|c|c|c|c|c|c|}
\hline $\begin{array}{c}\text { Organización } \\
\text { interna }\end{array}$ & $\begin{array}{c}\text { Un líder de } \\
\text { carácter social. }\end{array}$ & $\begin{array}{l}\text { Un líder de } \\
\text { carácter } \\
\text { comercial. }\end{array}$ & $\begin{array}{l}\text { Un líder de } \\
\text { carácter social. }\end{array}$ & $\begin{array}{l}\text { Un líder de } \\
\text { carácter } \\
\text { comercial. }\end{array}$ & $\begin{array}{c}\text { Un líder de } \\
\text { carácter social. }\end{array}$ & $\begin{array}{l}\text { Un líder de } \\
\text { carácter } \\
\text { comercial. }\end{array}$ \\
\hline $\begin{array}{l}\text { Indicadores } \\
\text { económicos }\end{array}$ & $\begin{array}{c}\text { VAN: } \\
\text { \$495.376.958, } \\
\text { Utilidad anual } \\
\text { por pescador: } \\
\text { \$991.610, Razón } \\
\text { C/I: } 24,2 \% .\end{array}$ & $\begin{array}{c}\text { VAN: } \\
\$ 1.677 .927 .992, \\
\text { Utilidad anual } \\
\text { por pescador: } \\
\text { \$1.877.542, Razón } \\
\text { C/I: } 42,3 \% \text {. }\end{array}$ & $\begin{array}{c}\text { VAN: } \\
\$ 245.677 .878, \\
\text { Utilidad anual } \\
\text { por pescador: } \\
\text { \$245.864, Razón } \\
\text { C/I: } 7,4 \% \text {. }\end{array}$ & $\begin{array}{c}\text { VAN: } \\
\text { \$398.483.549, } \\
\text { Utilidad anual } \\
\text { por pescador: } \\
\text { \$686.673, Razón } \\
\text { C/I: } 60,8 \% \text {. }\end{array}$ & $\begin{array}{c}\text { VAN: } \\
\text { \$1.592.128.418, } \\
\text { Utilidad anual } \\
\text { por pescador: } \\
\text { \$940.388, Razón } \\
\text { C/I: } 52,7 \% \text {. }\end{array}$ & $\begin{array}{l}\text { VAN: } \\
\$ 905.924 .113 \text {, } \\
\text { Utilidad anual } \\
\text { por pescador: } \\
\text { \$630.609, Razón } \\
\text { C/I: } 52,2 \%\end{array}$ \\
\hline
\end{tabular}

(Censo de Población y Vivienda, 2002), acceso a escolaridad (según Censo Nacional Pesquero y Acuicultor, 2008-2009), infraestructura, actividades relevantes, principales recursos pesqueros y un descriptor de organización interna (todos determinados mediante trabajo etnográfico), junto a indicadores económicos cuyo cálculo se describe en el texto. Donde, AMERB son Áreas de Manejo y Explotación de Recursos Bentónicos). Se entiende por líder de carácter social cuando la gestión realizada aborda la contingencia local en distintos ámbitos de la cotidianidad, contraria a la dirigencia que cumple una función más comercial que social.

\section{Levantamiento y recolección de información}

El trabajo en terreno consideró el levantamiento y generación de información cualitativa y cuantitativa, mediante: i) la aplicación de entrevistas en profundidad a actores claves de cada caleta (entre marzo 2008 y marzo 2011), con el objetivo de identificar y profundizar aspectos relativos a las prácticas cotidianas, productivas y la organización dentro de los modos de vida de los pescadores artesanales; ii) la realización de una encuesta bioeconómica aplicada a pescadores artesanales pertenecientes y no pertenecientes a sindicatos pesquero-artesanales, con el fin de estimar la estructura de costos de la pesquería, los días de extracción al año, las capturas obtenidas en cada faena, las artes de pesca utilizadas y los canales de comercialización de productos, entre otros aspectos económico-productivos. Ambos instrumentos fueron diseñados por el equipo de investigación y testeados con especialistas, luego fueron aplicados a nivel piloto en dos caletas, reajustando los ítems, para finalmente ser aplicados en cada caleta. Esta información contribuyó a distinguir las principales variables que inciden en la complejidad territorial asociadas a los procesos productivos de cada caleta. Las entrevistas y encuestas bioeconómicas solicitaron la misma información a pescadores y actores claves, solo variando según el tipo de pesquerías y la presencia de concesiones de acuicultura y/o áreas de manejo y explotación de recursos bentónicos. La selección de informantes fue realizada de la siguiente forma: i) para las entrevistas la muestra fue no probabilista, se buscaron pescadores y pescadoras mayores de 40 años y que vivían a lo menos 10 años en la caleta; el número de entrevistados se determinó a través del nivel de saturación, el cual se estableció entre 9 y 11 entrevistados. ii) Para las encuestas bioeconómicas el procedimiento fue similar, solo que se privilegió pescadores y pescadoras pertenecientes a distintos grupos etarios. Así, en cada caleta se contactaron al menos 12 pescadores hombres y mujeres: tres mayores de 65 años, tres entre 45 y 64 años, tres entre 30 y 45 años, 3 entre 18 y 29 años de edad.

En paralelo, se realizó una pesquisa a nivel bibliográfico y la revisión de las bases de datos del Servicio Nacional de Pesca (Sernapesca), la Subsecretaría de Pesca (Subpesca), el Censo Pesquero y Acuicultor 2008-2009 y el Censo de Población 2002, cuya información fue extraída mediante el uso del software REDATAM $^{\odot}$ utilizando herramientas de conteo y lista por área. Para la evaluación directa del desempeño económico de cada caleta, se eligieron como principales indicadores el VAN (valor presente 
neto), la utilidad anual promedio por pescador y la razón costo-beneficio (C/I). Para al cálculo del VAN se utilizó una tasa de descuento del 6\% (Mideplan) y un período de análisis de 10 años. No se consideraron las posibles inversiones realizadas a futuro, debido a la falta de información. La ganancia para cada ańo se supuso constante y se obtuvo a partir de la ganancia mensual estimada por cada pescador en el Censo Pesquero y Acuicultor de 2009. Para el cálculo de la utilidad anual por pescador, se restó a la ganancia promedio la depreciación de los botes. Esta fue calculada mediante una estimación del costo de cinco tipos de bote con igual vida útil (16 años). A partir de los costos y utilidades declarados por los pescadores para el año 2009, se obtuvieron los ingresos anuales promedio por pescador (ingresos $=$ costos + ganancia). Luego se calculó $\mathrm{C} / \mathrm{I}$ como la división entre los costos e ingresos anuales, expresándolo como porcentaje. Adicionalmente, se desarrolló un análisis de sensibilidad con el objetivo de determinar la variación porcentual de la utilidad en cada caleta, frente a una variación del precio de sus principales recursos pesqueros y del combustible utilizado para las faenas. Para esto, fue necesario obtener el desembarque anual promedio de cada recurso. Si bien se disponía de una base de datos de Sernapesca con los desembarques y precios para cada especie y caleta entre los años 2000 y 2009, se optó por no utilizarlos directamente debido a que, como se pudo constatar en las encuestas bioeconómicas, las extracciones reales son mayores a las declaradas, debido al efecto de la pesca ilegal o no-declarada. No obstante, se asumió que esta base de datos entregó una buena aproximación de cuáles son los principales recursos pesqueros explotados en cada caleta y la proporción que cada uno aporta al desembarque total de la caleta. Por lo tanto, a partir de esta base de datos se determinaron las cinco especies hidrobiológicas que generan mayores ingresos en cada caleta. Luego, con los datos de desembarques históricos de cada una de estas especies, se determinó el porcentaje que aporta cada una al desembarque total de la caleta, generando así una distribución de desembarques. Teniendo esta distribución porcentual, los ingresos totales de la caleta y los precios de cada especie (a partir de la encuesta bioeconómica y datos de Sernapesca), se infirió la cantidad real del desembarque anual para cada recurso hidrobiológico.

\section{Construcción del ICT}

Parte de la estrategia metodológica se sustenta en la utilización de técnicas avanzadas de sistematización y análisis de información. Dentro de estas destaca el Análisis Multicriterio (AMC) como herramienta para el diseńo y construcción del ICT, convocado por medio de un Panel de Expertos de carácter interdisciplinario (antropólogos, geógrafos y biólogos) y que ha procurado relevar distintas dimensiones de las relaciones socioambientales en las caletas de pesca artesanal. Aquí, el AMC se ha utilizado como la base metodológica que permite el análisis de distintas cualidades de las caletas de pescadores artesanales para aunar en el análisis variables cualitativas y cuantitativas, posibilitando ponderar su grado de importancia a la hora de dimensionar cuál de estas caletas tiene condiciones de complejidad territorial más altas, y ligadas a mejores condiciones de sustentabilidad. El AMC se erige como una metodología de integración de variables para la resolución de problemas complejos, con una gran cantidad de variables y cualidades particulares (Moreno-Jiménez et al., 1998; Munda, 2004; Aznar et al., 2007).

El ICT corresponde a un índice cuantitativo que pretende dar cuenta de los niveles de sustentabilidad de cada caleta, construido a partir de una estructura de variables y criterios relevantes para la investigación, que fueron definidos mediante el ejercicio interdisciplinario antes descrito, y complementados mediante la ponderación de criterios y subcriterios por medio del software EXPERT CHOICE ${ }^{\oplus}$ (Tabla 2). Así, a través de su herramienta "comparación de importancia relativa" apoyó la ponderación de los criterios a ser considerados en el ICT, ponderaciones que el mismo programa se encarga de vigilar a través del cálculo de una tasa de inconsistencia, proceso matemático realizado paralelamente a la ponderación de criterios y subcriterios, que indica si las ponderaciones siguen una lógica o se están haciendo al azar (Ishizaka y Labib, 2009). Por medio de este proceso, se obtuvo un indicador de ponderación numérico a partir de la división de cada unidad entre sus componentes, cuyos criterios y subcriterios sumaron el valor de 1 (uno). Los criterios seleccionados fueron ordenados priorizando las alternativas, de acuerdo a ponderaciones de mayor a menor. Para estandarizar la información recogida y descrita, se 
calificó cada subcriterio de la siguiente forma: Rango 1: Bajo (Grado de organización mínimo), Rango 2: Medio (Grado de organización insuficiente), Rango 3: Alto (Grado de organización suficiente).

Luego de clasificar cada criterio, el siguiente paso fue la operacionalización de cada subcriterio, obteniéndose un índice promedio posible de correlacionar con los índices de otros criterios y que finalmente permiten obtener un único índice que sintetiza la complejidad territorial, y cuyos valores dan cuenta de las diversas capacidades y condiciones de sustentabilidad de cada caleta.

Junto con la construcción de este índice, se optó por la utilización del Análisis de Componentes Principales (ACP) como herramienta para la síntesis de la amplia información generada. La idea central del ACP es conseguir la simplificación de un conjunto de datos, generalmente cuantitativos, procedentes de un conjunto de variables interrelacionadas (Almenara-
Barrios et al., 2002). Para seleccionar las variables clasificadas se utilizó el criterio del valor propio (Eigenvalue), que corresponde a la cantidad de varianza explicada y acumulada de cada componente o factor. Por esta razón solo se consideraron valores propios mayores a uno, cuando generalmente se seleccionan los tres primeros factores, ya que explican la mayor proporción de la varianza (>70\%). Para la representación gráfica se utilizó un análisis Biplot de correlación (variables continuas o discretas) cuya finalidad es aproximar los datos originales y realizar un análisis simultáneo de las relaciones entre individuos y/o variables. Se analizaron las dieciséis variables clasificadas en cuatro criterios, para el total de caletas. Se realizaron tres análisis de componentes principales: uno para las caletas de mar interior, otro para las caletas del mar abierto y uno para el total de las caletas. Para ello se utilizó el programa XLSTAT 2013. Todos los $\mathrm{CP}$ fueron estandarizaron con rotación VARIMAX (rotación ortogonal que minimiza el número de variables que tiene saturaciones altas en cada factor).

Tabla 2. Criterios y subcriterios considerados en el ICT. Indica además su ponderación y siglas.

\begin{tabular}{|c|c|c|c|}
\hline Criterios & Subcriterios & Ponderación & Sigla \\
\hline \multirow{4}{*}{$\begin{array}{l}\text { Nivel organizacional } \\
\text { Participación-Gestión }\end{array}$} & Figura de los líderes & 0,045 & FL \\
\hline & Objetivos logrados & 0,565 & $\mathrm{OL}$ \\
\hline & Forma de tomar decisiones & 0,099 & FTD \\
\hline & Nivel de compromiso & 0,291 & NC \\
\hline \multirow{5}{*}{ Principales problemas en la caleta } & Accesibilidad & 0,211 & A \\
\hline & Acceso a las TIC & 0,027 & AT \\
\hline & Nivel de escolaridad & 0,202 & NE \\
\hline & Ocupación & 0,356 & $\mathrm{O}$ \\
\hline & Infraestructura y equipamiento & 0,204 & IE \\
\hline \multirow{3}{*}{ Economía pesquero artesanal } & Economía local & 0,726 & EL \\
\hline & Rentabilidad de las pesquerías & 0,198 & $\mathrm{RP}$ \\
\hline & Comercialización de recursos & 0,076 & CR \\
\hline \multirow{4}{*}{ Recursos pesqueros } & Recursos extraídos & 0,139 & $\mathrm{RE}$ \\
\hline & Esfuerzo pesquero & 0,195 & EP \\
\hline & Incorporación conocimiento científico & 0,510 & ICC \\
\hline & Características físico-naturales & 0,157 & CFN \\
\hline
\end{tabular}




\section{Resultados}

\section{Índice de complejidad territorial}

En la Tabla 3 se entregan los resultados del ICT con las ponderaciones de criterios y subcriterios para cada una de las caletas estudiadas. En general, las caletas de Cucao y Puntilla Pichicolo obtuvieron los menores valores del ICT, mientras que caleta El Manzano destacó por presentar el valor más alto (Tabla 3). Con base en los valores máximos y mínimos del ICT, fue posible identificar de forma tentativa las debilidades y fortalezas de cada caleta. Así, en la caleta de Cucao su fortaleza fue la "Accesibilidad" y sus debilidades la "Incorporación del conocimiento científico" y "Comercialización de los Recursos". En caleta Puntilla Pichicolo su fortaleza fueron las "Características físico-naturales" y su debilidad la "Infraestructura y equipamiento". En la caleta de Tenaún su fortaleza fue el "Acceso a las TIC'S” y debilidad la "Figura de los Líderes”. En la caleta de Puñihuil su fortaleza fueron el "Esfuerzo Pesquero" e "Incorporación del conocimiento científico", y su debilidad la "Forma de tomar decisiones". En la caleta de Guabún su fortaleza fueron los "Objetivos Logrados" y la "Figura de Los Líderes", mientras que su debilidad los "Recursos Extraídos" e "Infraestructura y equipamiento". En la caleta de El Manzano, por último, su fortaleza fue la "Forma de tomar decisiones" y debilidad la "Economía Local".

Tabla 3. Resultados del Índice de Complejidad Territorial (ICT) para seis caletas del archipiélago de Chiloé.

\begin{tabular}{|c|c|c|c|c|c|c|c|c|}
\hline \multirow[b]{2}{*}{ Criterios } & \multirow[b]{2}{*}{ Subcriterios } & \multirow[b]{2}{*}{ Pond } & \multicolumn{6}{|c|}{ Caletas de pesca artesanal } \\
\hline & & & Cucao & Puntilla & Tenaún & Puñihuil & Guabún & El manzano \\
\hline \multirow{4}{*}{$\begin{array}{c}\text { Explotación } \\
\text { pesquero- } \\
\text { artesanal. } \\
\text { Ponderación: } \\
0,528\end{array}$} & $\begin{array}{l}\text { Recursos } \\
\text { extraídos }\end{array}$ & 0,139 & 0,095 & 0,104 & 0,064 & 0,282 & 0,048 & 0,406 \\
\hline & Esfuerzo pesquero & 0,195 & 0,033 & 0,159 & 0,07 & 0,435 & 0,052 & 0,252 \\
\hline & $\begin{array}{c}\text { Incorporación } \\
\text { del conocimiento } \\
\text { científico }\end{array}$ & 0,51 & 0,029 & 0,143 & 0,059 & 0,435 & 0,085 & 0,25 \\
\hline & $\begin{array}{l}\text { Características } \\
\text { físico-naturales }\end{array}$ & 0,157 & 0,037 & 0,313 & 0,163 & 0,057 & 0,084 & 0,346 \\
\hline \multirow{3}{*}{$\begin{array}{c}\text { Economía } \\
\text { pesquero- } \\
\text { artesanal. } \\
\text { Ponderación: } \\
0,240\end{array}$} & Economía local & 0,726 & 0,3 & 0,08 & 0,121 & 0,04 & 0,301 & 0,158 \\
\hline & $\begin{array}{l}\text { Rentabilidad de } \\
\text { las pesquerías }\end{array}$ & 0,198 & 0,041 & 0,12 & 0,126 & 0,345 & 0,189 & 0,179 \\
\hline & $\begin{array}{c}\text { Comercialización } \\
\text { de los recursos }\end{array}$ & 0,076 & 0,029 & 0,215 & 0,053 & 0,197 & 0,12 & 0,387 \\
\hline \multirow{5}{*}{$\begin{array}{c}\text { Principales } \\
\text { problemas } \\
\text { de la caleta. } \\
\text { Ponderación: } \\
0,051\end{array}$} & Accesibilidad & 0,211 & 0,274 & 0,062 & 0,099 & 0,077 & 0,266 & 0,223 \\
\hline & $\begin{array}{c}\text { Acceso a las } \\
\text { TIC'S }\end{array}$ & 0,027 & 0,04 & 0,032 & 0,417 & 0,051 & 0,081 & 0,379 \\
\hline & $\begin{array}{c}\text { Nivel de } \\
\text { escolaridad }\end{array}$ & 0,202 & 0,146 & 0,072 & 0,378 & 0,077 & 0,075 & 0,251 \\
\hline & Ocupación & 0,356 & 0,053 & 0,1 & 0,047 & 0,418 & 0,191 & 0,19 \\
\hline & $\begin{array}{c}\text { Infraestructura y } \\
\text { equipamiento }\end{array}$ & 0,204 & 0,127 & 0,028 & 0,337 & 0,044 & 0,049 & 0,414 \\
\hline \multirow{4}{*}{$\begin{array}{c}\text { Nivel de } \\
\text { organización } \\
\text { de los } \\
\text { pescadores } \\
\text { artesanales. } \\
\text { Ponderación: } \\
0,182\end{array}$} & $\begin{array}{c}\text { Figura de los } \\
\text { líderes }\end{array}$ & 0,045 & 0,092 & 0,039 & 0,031 & 0,049 & 0,416 & 0,372 \\
\hline & $\begin{array}{l}\text { Objetivos } \\
\text { logrados }\end{array}$ & 0,565 & 0,035 & 0,051 & 0,062 & 0,061 & 0,44 & 0,351 \\
\hline & $\begin{array}{c}\text { Forma de tomar } \\
\text { decisiones }\end{array}$ & 0,099 & 0,077 & 0,055 & 0,042 & 0,027 & 0,355 & 0,443 \\
\hline & $\begin{array}{c}\text { Nivel de } \\
\text { compromiso }\end{array}$ & 0,291 & 0,08 & 0,059 & 0,037 & 0,032 & 0,394 & 0,399 \\
\hline \multicolumn{3}{|c|}{ Total } & 1.488 & 1,632 & 2,106 & 2,627 & 3,146 & 5 \\
\hline
\end{tabular}


Luego de cruzar los resultados del ICT con el ACP, se observó que las caletas ubicadas en mar abierto presentan dos factores con valores propios mayores a 1 , identificados como Factor $1(57,31 \%$ de la variabilidad explicada) constituido por los subcriterios de los criterios "Explotación pesquero artesanal" y "Economía pesquero artesanal", y el Factor 2 (42,68\% de la variabilidad explicada) correspondiente a "Niveles de organización de los pescadores artesanales". Los resultados de la rotación Varimax indican que ambos criterios corresponden a los que presentan mayor correlación para explicar la situación de estas caletas (Tabla 4). Por otra parte, las caletas ubicadas en el mar interior presentaron el Factor 1 (74,42\% de la variabilidad explicada), constituido por los subcriterios de los criterios "Explotación pesquero-artesanal" y "Niveles de organización de los pescadores artesanales", y el Factor $2(25,57 \%$ de la variabilidad explicada) correspondiente a "Principales problemas de la caleta". Para el total de las caletas, los resultados muestran una correspondencia con lo anteriormente señalado, donde los subcriterios de los criterios "Explotación pesquero artesanal" (F1, 34,17\% de la variabilidad explicada) y "Niveles de organización de los pescadores artesanales" (F2, 33,41\% de la variabilidad explicada) presentaron la mayor correlación para la explicación del desempeńo de las caletas (Tabla 4).

Tabla 4. Correlaciones entre los subcriterios y factores tras rotación Varimax.

\begin{tabular}{|c|c|c|c|c|c|c|c|c|}
\hline \multirow{2}{*}{ Criterios } & \multirow{2}{*}{ Subcriterios } & \multicolumn{2}{|c|}{ Mar abierto } & \multicolumn{2}{|c|}{ Mar interior } & \multicolumn{2}{|c|}{ Total } & \\
\hline & & $\mathrm{F} 1$ & $\mathrm{~F} 2$ & $\mathrm{~F} 1$ & $\mathrm{~F} 2$ & $\mathrm{~F} 1$ & $\mathrm{~F} 2$ & \\
\hline \multirow{4}{*}{$\begin{array}{c}\text { Explotación } \\
\text { artesanal }\end{array}$} & $\mathrm{RE}$ & 0,853 & $-0,522$ & 0,958 & 0,285 & 0,775 & 0,242 & \\
\hline & EP & 0,950 & $-0,311$ & 0,993 & $-0,114$ & 0,972 & $-0,177$ & \\
\hline & ICC & 0,974 & $-0,228$ & 0,998 & $-0,057$ & 0,981 & $-0,094$ & \\
\hline & CFN & 0,269 & 0,963 & $\mathbf{0 , 8 8 7}$ & $-0,462$ & 0,224 & 0,122 & \\
\hline \multirow{3}{*}{$\begin{array}{l}\text { Economía } \\
\text { pesquero- } \\
\text { artesanal }\end{array}$} & EL & $-0,935$ & 0,353 & 0,582 & 0,813 & $-0,683$ & 0,629 & \\
\hline & $\mathrm{RP}$ & 0,989 & 0,150 & 0,883 & 0,470 & 0,883 & 0,014 & \\
\hline & CR & $\mathbf{0 , 9 7 7}$ & 0,212 & 0,994 & $-0,109$ & 0,721 & 0,383 & \\
\hline \multirow{5}{*}{$\begin{array}{c}\text { Principales } \\
\text { problemas de } \\
\text { la caleta }\end{array}$} & A & $-0,949$ & 0,317 & 0,815 & 0,579 & $-0,436$ & 0,784 & \\
\hline & AT & 0,096 & 0,995 & 0,037 & 0,999 & $-0,044$ & 0,097 & \\
\hline & $\mathrm{NE}$ & $-0,756$ & $-0,655$ & $-0,294$ & 0,956 & $-0,290$ & $-0,145$ & 0,858 \\
\hline & $\mathrm{O}$ & 1,000 & 0,026 & 1,000 & 0,021 & 0,890 & 0,081 & $-0,349$ \\
\hline & IE & $-0,805$ & $-0,594$ & 0,311 & 0,950 & $-0,061$ & 0,182 & 0,934 \\
\hline \multirow{4}{*}{$\begin{array}{c}\text { Nivel de la } \\
\text { organización } \\
\text { de los } \\
\text { pescadores }\end{array}$} & FL & $-0,270$ & 0,963 & 0,930 & 0,367 & 0,056 & 0,989 & 0,064 \\
\hline & $\mathrm{OL}$ & $-0,108$ & 0,994 & 0,909 & 0,416 & 0,095 & 0,948 & 0,071 \\
\hline & FTD & $-0,303$ & 0,953 & 0,933 & 0,360 & 0,111 & 0,954 & 0,271 \\
\hline & NC & $-0,284$ & 0,959 & 0,942 & 0,336 & 0,069 & 0,979 & 0,155 \\
\hline
\end{tabular}

Nota: Para las siglas de los subcriterios, ver Tabla 2. 
El análisis Biplot permitió observar que las caletas ubicadas en mar abierto son únicas y en general no comparten características comunes (Figura 2). Por ejemplo, la caleta de Puñihuil se caracterizó por tener una alta correlación con el criterio de "Explotación pesquero artesanal" (D1), mientras que la de Guabún presentó alta correlación con el criterio "Niveles de organización de los pescadores artesa- nales" (D2) (Figura 2). Por otra parte, para las tres caletas ubicadas en el mar interior se observó una alta correlación de la mayoría de las variables (subcriterios) con la caleta El Manzano (Figura 3). El mismo análisis aplicado al total de caletas estudiadas permitió observar que El Manzano comparte cuatro variables con Guabún y otras cinco con Puñihuil (Figura 4).

Biplot (ejes D1 y D2: 100,00 \%)

tras rotación Varimax

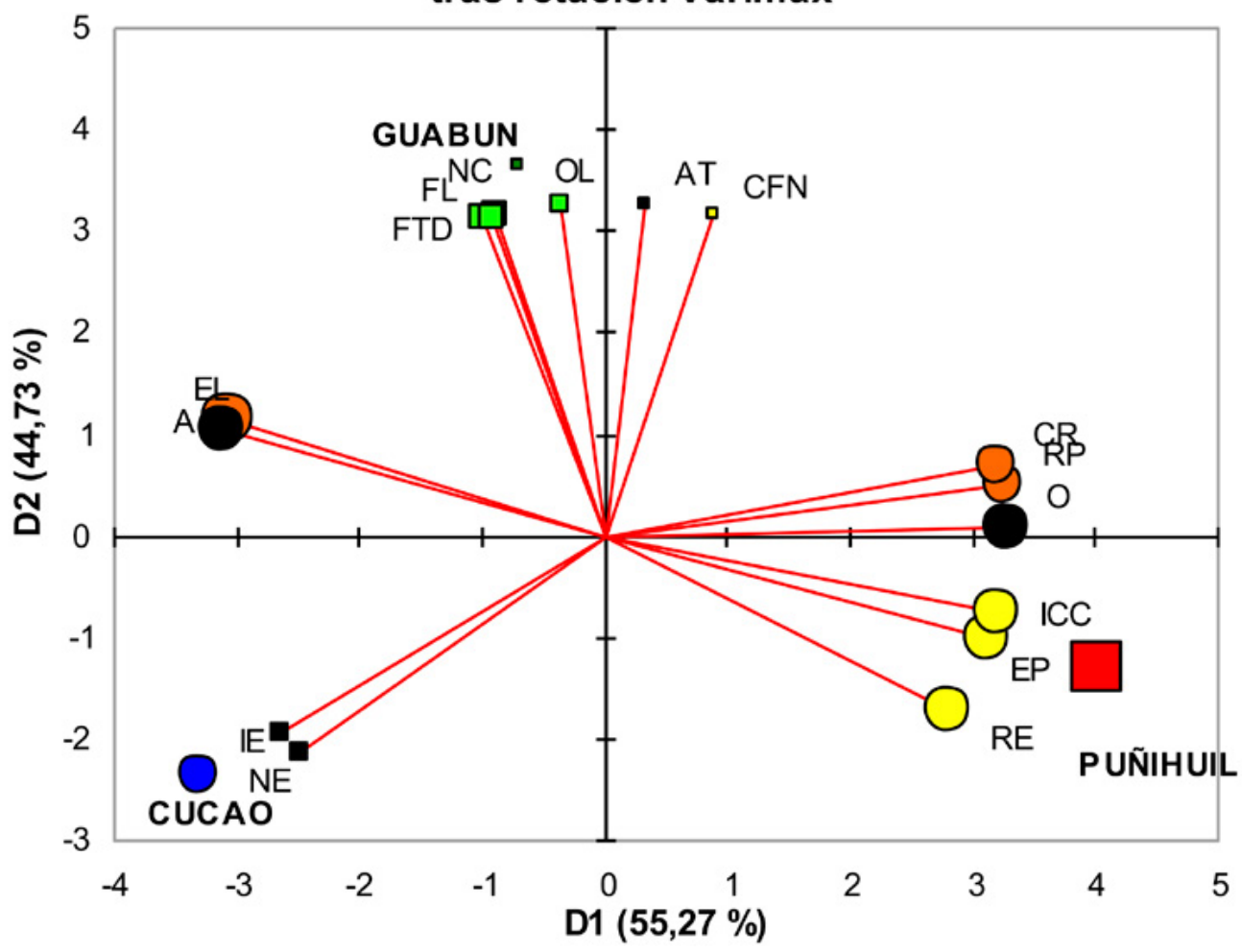

Figura 2. Resultados del Biplot para las tres caletas ubicadas en el mar abierto. Para detalles, ver el texto y Tabla 2. 


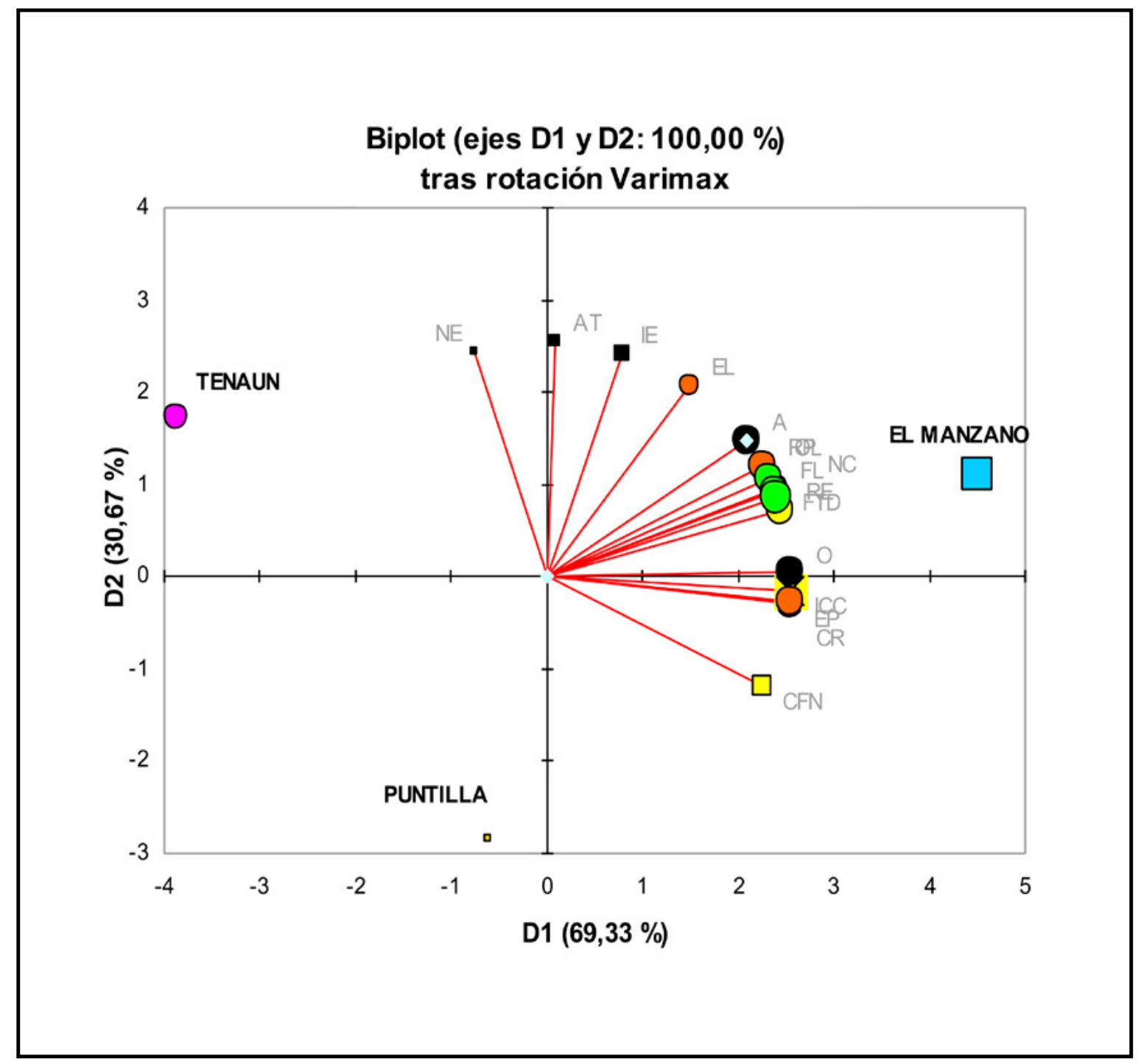

Figura 3. Resultados del Biplot para las tres caletas ubicadas en el mar interior. Para detalles, ver el texto y Tabla 2. 


\section{Biplot (ejes D1 y D2: 65,68 \%) tras rotación Varimax}

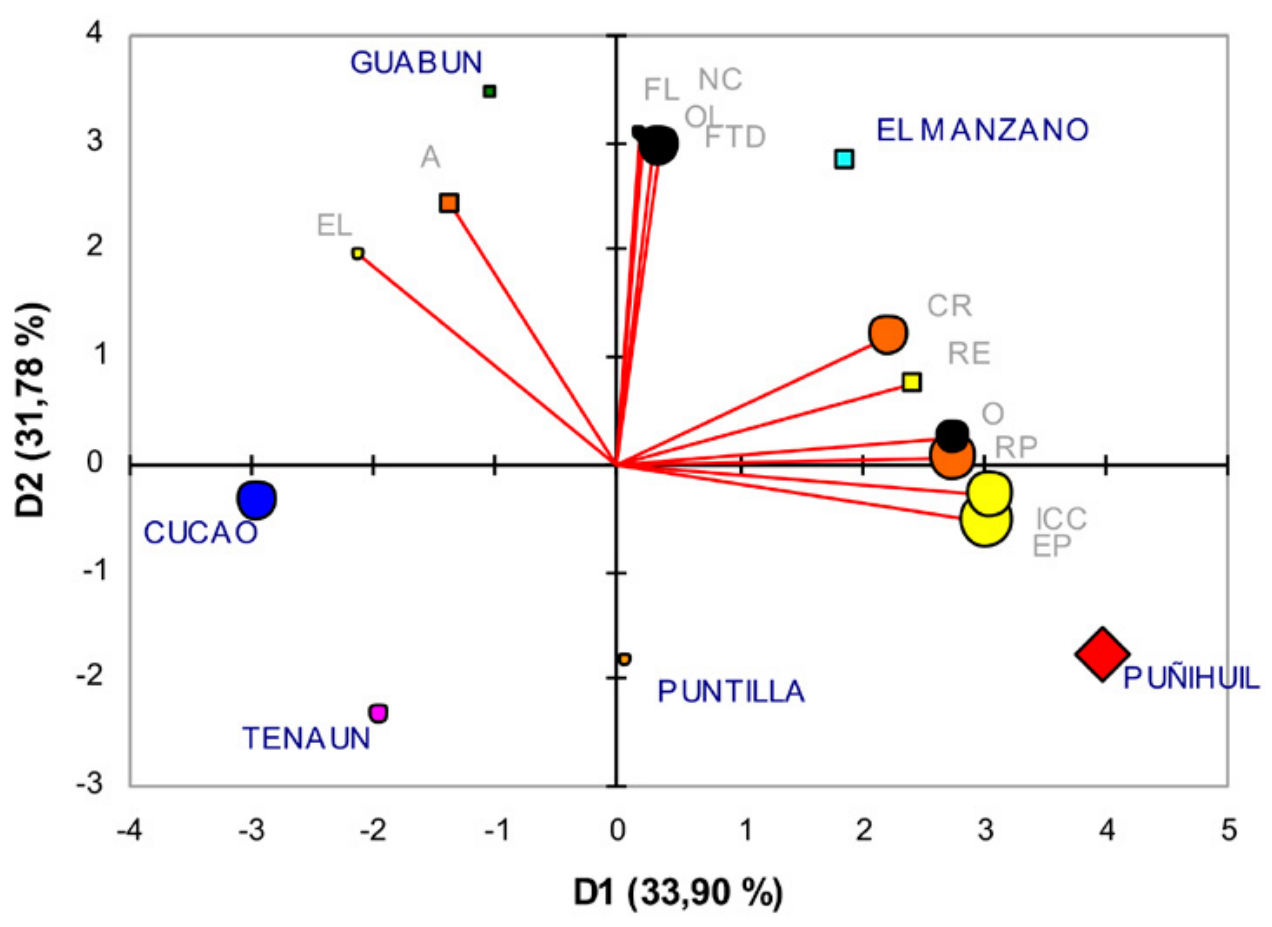

Figura 4. Resultados del Biplot para el total de caletas estudiadas. Para detalles, ver el texto y Tabla 2.

\section{Discusión}

El ICT permitió distinguir las capacidades de resiliencia y sustentabilidad de cada caleta, entregando indicadores sintéticos que permiten la comparación de estas cualidades socioecológicas complejas. En primer lugar interesa destacar que el ICT permite clasificar desempeños en materia de resiliencia y sustentabilidad, en tanto cualidades de los sistemas socioecológicos (Janssen et al. 2006; Folke et al. 2002). En este caso, el ICT refiere a los procesos de adaptación y vinculación al medio ambiente para cada uno de los criterios y subcriterios definidos y ponderados. En otras palabras, sintetiza la coevolución socioambiental en cada uno de los subcriterios y de manera global. Así, caleta El Manzano posee el mayor valor de ICT y se presenta como el asentamiento con mejor evaluación. Por lo cual, posee mayor cercanía a un estado relativo o referente de resiliencia y sustentabilidad. Esto porque obtuvo la mejor evaluación en gran parte de los subcriterios identificados y ponderados, como los de Recurso extraído, Esfuerzo pesquero, Características físiconaturales, Comercialización de recursos, Infraestructura y equipamiento, Objetivos logrados, Forma de tomar decisiones y Nivel de compromiso. 
No obstante, que caleta El Manzano presente un ICT $=5$ no significa que se trate de una caleta sustentable ni resiliente, puesto que estas cualidades son antes procesos más que estados. Sin embargo, permite identificar la posición relacional de la caleta dentro de la muestra completa, indicando que posee condiciones más favorables para el desarrollo sostenible de la actividad pesquero-artesanal que el resto de ellas. También corresponde señalar que el ICT, por presentarse como un índice intermuestral, no tiene la capacidad de ser comparable con otras muestras, aunque se haya seguido la misma metodología de análisis. No obstante, el ICT es una herramienta útil para el análisis de los territorios, ya que su complejidad aumenta al momento de estudiar lo micro, lo local (Santos, 2000).

En cuanto al papel de la localización, según el ICT la ubicación de las caletas en el mar abierto o mar interior no parece ser una variable relevante para la evaluación general de la muestra. En este sentido, los resultados del cálculo del ICT dejan en evidencia de qué modo la aplicación de enfoques deterministas en la geografía fracasa ante factores culturales, sociales y económicos de un territorio (Santos, 1990). De esta manera, el estudio de la complejidad de las caletas de pescadores artesanales debiera partir de la noción de que las condiciones geográficoambientales no pueden tener un peso mayor, a la hora de buscar sus forzantes o variables explicativas del contexto social.

Desde el siglo pasado, el análisis internacional sobre el desarrollo de la pesca artesanal indica la necesidad de impulsar visiones interdisciplinarias y multidimensionales, como así también reconoce tres grandes fases para el ordenamiento territorial: análisis, planificación y gestión (Hildenbrand, 1996). De esto, la operatividad del ICT permite sintetizar las relaciones socioambientales al identificar los componentes de mayor relevancia y entregar orientaciones para el desarrollo de intervenciones destinadas al fomento de capacidades de resiliencia y sustentabilidad del territorio. Por lo cual, el ICT apoya la planificación territorial en el sentido de que hace posible focalizar recursos públicos de manera diferenciada $y$ según las necesidades territoriales de cada caleta de pesca artesanal, junto con permitir realizar acciones de seguimiento a los procesos encaminados.
Diversos estudios se han cuestionado cómo diseñar e implementar estrategias que permitan mejorar la calidad de vida de pescadores artesanales y el manejo local de sus recursos, como también hacer frente a la crisis por sobreexplotación de la biodiversidad marina y costera (García-Allut, 1999, 2003; Pauly et al., 2006). Los resultados del ICT muestran diferencias entre diversidades internas de las caletas estudiadas, permitiendo focalizar estrategias de mejoramiento de gestión que resulten de importancia para el desarrollo del sector pesquero artesanal. Aportando a distanciar la gestión territorial de su habitual sesgo hacia el mercado, evitando ver la pesca artesanal como sistemas exclusivamente económicos, cuyos elementos son susceptibles de privatización y mercantilización (Suárez de Vivero et al., 2008), sin considerar sus aspectos culturales y socioambientales específicos.

El resultado global del ICT permite la reformulación de nuestro modelo inicial, excluyendo los subcriterios identificados como de menor relevancia. Así también, hace posible identificar focos prioritarios para la mejora de la gestión de estos asentamientos costeros, guiando los procesos de intervención de políticas públicas mediante el manejo de los criterios relevantes. De esta manera, la combinación del ICT en tanto herramienta para la medición e interpretación de los desempeños de sustentabilidad y resiliencia, más la síntesis de correlaciones ofrecida por ACP, permiten derivar en un modelo de análisis de profunda ventaja aplicada a campos académicos y de gestión costera.

\section{Consideraciones finales}

Las orientaciones que el índice entrega permiten mapear una gran cantidad de territorios costeros y establecer puntos de interés en donde poder comenzar a realizar dichas estrategias de vinculación entre científicos y pescadores. Esto se fundamenta en que el ICT pone de manifiesto aquellos territorios donde el arraigo territorial, entendido como la producción de territorios vividos por parte de pescadores y pescadoras (Ther-Ríos, 2006, 2008, 2011) ha generado una construcción social de la biodiversidad, ha identificado sectores del territorio con sus ciclos naturales, ha conocido las relaciones de alimentación 
con las especies y sobre la base de ello ha generado un sistema de extracción de recursos y un sistema de comercialización hacia diversos destinos. Su aplicabilidad a contextos socioterritoriales variados deviene del hecho de erigirse como una metodología estandarizada, pero en cuyas etapas se tiene como premisa la inclusión de los aspectos, tanto cuantitativos como cualitativos, característicos de los territorios a analizar. Además, el ICT también se manifiesta como una metodología integradora de distintos tipos de información (primaria y secundaria) que bajo otras metodologías sería difícil de aunar.

Como ya se mencionó, el concepto de complejidad alude al aumento de diferenciación de partes de un sistema determinado (Tainter, 1995), de lo cual el ICT permite ver diferencias en las caletas de mar abierto y mar interior y entre todas ellas, dando cuenta de cómo los espacios esconden, en sus escalas micro, interacciones que no son posibles de observar en una escala mayor (ya sea comunal o regional), pero que pueden advertir acerca de los procesos que impactan en las escalas superiores (Santos, 2000). Con todo ello, el ICT permite identificar aquellos territorios en donde a partir del complejo vínculo entre sociedad-naturaleza, los asentamientos costeros demuestran una mayor vinculación y diálogo entre biodiversidad y sociodiversidad. Esta vinculación sociedad-naturaleza presente en los distintos territorios cobra mayor fuerza en los pescadores artesanales, toda vez que la apropiación, uso y manejo de los recursos pesqueros y las apropiaciones espaciales devenidas de estas prácticas (entendidas estas como prácticas cotidianas cargadas de aspectos culturales) están indisolublemente ligadas a sentimientos de identidad (Claval, 2002). Lo que involucra un proceso de aprendizaje ecológico en torno al cuidado de los recursos pesquero-artesanales, conduciendo al pescador artesanal a la necesidad de interpretar el entorno natural con la finalidad de crear las condiciones óptimas para posibilitar el acceso, así como la actuación sobre él. Por lo tanto, supone una fase fundamental del proceso de adaptación, generando conocimiento y cultura en su intento por "dar explicaciones a cosas y a hechos" (García Allut, 1999). En fin, complejizar esta relación con el medio en búsqueda de sustentabilidad socioambiental. En este sentido, el índice se vincula a una antropolítica de la diversidad socioecológica, que permite avanzar en instancias de participación (Alcalá, 2003), gestión local de recursos (Barragán et al., 2005), empoderamiento de comunidades y gobernanza de bienes comunes (Berkes y Folke, 1998; Ostrom, 2000) y concepciones de territorio ligadas a aspectos socioculturales y prácticas cotidianas en contraposición a las dimensiones administrativas del mismo (Santos, 1990; Santos, 2000; Claval, 2002).

\section{Agradecimientos}

Se agradece a los pescadores artesanales del archipiélago de Chiloé por la confianza que han tenido con nuestro equipo; asimismo los aportes del equipo de investigación del Programa ATLAS de la ULAGOS, como también a CONICYT - Chile, quienes han aportado con nuestra investigación a través de los Proyectos FONDECYT 1080665 y 1121204.

\section{Referencias citadas}

Alcalá, G. (1999). Con el agua hasta los aparejos: pescadores y pesquerias en el Soconusco, Chiapas. Chiapas, México: Centro de Investigaciones y Estudios Superiores en Antropología Social.

Alcalá, G. (2003). Políticas Pesqueras en México: $1946-$ 2000. Contradicciones y Aciertos en la Planificación de la Pesca Nacional. México: El Colegio de México - Centro de Investigación Científica y de Educación Superior de Ensenada - El Colegio de Michoacán.

Almenara-Barrios, J., García-Ortega, C., González-Caballero, J. L. y Abellán-Hervás, M. J. (2002). Creación de índices de gestión hospitalaria mediante análisis de componentes principales. Salud pública de México, 44, 533-540.

Aznar, J., Guijarro, F. y Moreno-Jiménez, J. M. (2007). Valoración agraria multicriterio en un entorno con escasa información. Estudios de Economía Aplicada, 5, 549-572.

Barragán, J. M., Alvarado, C. y Castro, C. (2005). Hacia la Gestión Integrada de las Zonas Costeras en Chile. En Barragán, J. M. (Ed). La Gestión de Áreas Litorales en España y Latinoamérica pp. 109-136). Cádiz, España: Universidad de Cádiz. 
Barton, J. R. y Floysand, A. (2010). The political ecology of Chilean salmon aquaculture, 1982-2010: a trajectory from economic development to global sustainability. Global Environmental Change-Human and Policy Dimensions, 20, 739-752.

Béné, C., Macfadyen, G. y Allison, E. H. (2007). Increasing the contribution of small-scale fisheries to poverty alleviation and food security. Roma: FAO Technical Guidelines for Responsible Fisheries.

Berkes, F. (2008). La pesquería de pequeña escala: alternativa al manejo convencional de recursos. En Pinedo, D. y Soria, C. (Eds.). El manejo de las pesquerias en rios tropicales de Sudamérica (pp. 443-459). Bogotá: Mayol Ediciones.

Berkes, F. y Folke, C. (1998). Linking social and ecological systems for resilience and sustainability. En Berkes, F. y Folke, C. (Eds.). Linking Social and Ecological Systems: management practices and social mechanisms for building resilience (pp. 1-25). Cambridge: Cambridge University Press.

Berkes, F., Colding, J. y Folke, C. (Eds.) (2003). Navigating Social-Ecological Systems: Building Resilience for Complexity and Change. Cambridge: Cambridge University Press.

Bossel, H. (2001). Assessing viability and sustainability: a systems-based approach for deriving comprehensive indicator sets. Conservation Ecology, 5, 12.

Bustos, B. (2013). The ISA crisis in Los Lagos Chile: A failure of neoliberal environmental governance? Geoforum, 48, 196-206.

Claval, P. (2002). El enfoque cultural y las concepciones geográficas del espacio. Boletín de la AGE, 34, 21-39.

Constanza, R., Waigner, L., Folke, C. y Mäler, K. (1993). Modeling complex ecological economic systems: towards an evolutionary dynamic understanding of people and nature. BioScience, 43, 545-555.

Cormont, A., Siepel, H., Clement, J., Melman, T. C., Wallisdevries, M. F., Van Turnhout, C. A., Sparrius, L. B., Reemer, M., Biesmeijer, J. C., Berendse, F. y De Snoo, G. R. (2016). Landscape complexity and farmland biodiversity: evaluating the CAP target on natural elements. Journal for Nature Conservation, 30, 19-26.
Davies, J. L. (1972). Geographical variation in coastal development. New York: Longman Publishing Group.

Defeo, O., Castrejón, M., Ortega, L., Kuhn, A. M., Gutiérrez, N. L. y Castilla, J. C. (2013). Impacts of climate variability on Latin American small-scale fisheries. Ecology and Society, 18, 30.

FAO, Organización de las Naciones Unidas para la Alimentación y la Agricultura (2000). El estado mundial de la pesca y acuicultura 2000. Roma: FAO Fisheries Technical Paper.

Folke, C. (2006). Resilience: The emergence of a perspective or social-ecological systems analyses. Global Environmental Change, 16, 253-267.

Folke, C., Carpenter, S., Elmqvist, T., Guderson, L., Holling, S. y Walker, B. (2002). Resilience and sustainable development: building adaptive capacity in a world of transformation. AMBIO, 31, 437-440.

Fraser, E., Dougill, A., Mabee, W., Reed, M. y Mcalpine, P. (2006). Bottom up and top down: Analysis of participatory processes for sustainability indicator identification as a pathway to community empowerment and sustainable environmental management. Journal of Environmental Management, 78, 114-127.

Gajardo, C. y Ther-Ríos, F. (2011). Saberes y prácticas pesquero-artesanales: cotidianeidades y desarrollo en las caletas de Guabún y Puñihuil, Isla de Chiloé. Chungara. Revista de Antropología Chilena, 43, 589-605.

García-Allut, A. (1999). Compartición del conocimiento tradicional y científico para una gestión más adecuada de las pesquerías. Etnográfica, 3, 109-331.

Giraut, F. (2011). Bioregionalization and territorial complexity in the global South. Procedia-Social and Behavioral Sciences, 14, 49-52.

González, J. A., Montes, C., Rodríguez, J. y Tapia, W. (2008). Rethinking the Galapagos Islands as a complex social-ecological system: implications for conservation and management. Ecology and Society, 13, 13.

Graymore, L. M., Sipe, N. y Rickson, R. (2008). Regional Sustainability: How useful are current tools of sustainability assessment at the regional scale. Ecological Economics, 67, 362-372. 
Gristo, P., De Santa Ana, H., Martino, R., Tomasini, J. y Blánquez, N. (2015). Application of best practices and evaluation of the performance in health, safety and environment management for a large seismic programme offshore Uruguay. En SPE Latin American and Caribbean Health, Safety, Environment and Sustainability Conference. Bogotá: Society of Petroleum Engineers.

Gunderson, L. H. y Holling, , C. S. (Eds.) (2002). Panarchy: Understanding Transformations in Human and Natural Systems. Washington: Island Press.

Gunderson, L. H., Holling, C. S. y Light, S. (Eds.) (1995). Barriers and Bridges to the Renewal of Ecosystems and Institutions. New York: Columbia University Press.

Hall, S. J. (2011). Climate change and other external drivers in small-scale fisheries: practical steps for responding. En Pomeroy, R. S. y Andrew, N. L. (Eds.). Small scale fisheries management: frameworks and approaches for the developing world (pp. 132-159). Cambridge, MA: CAB International.

Haughton, G. (1999). Environmental justice and the sustainable city. Journal of Planning Education and Research, $18,233-243$.

Hildenbrand, A. (1996). Politica de ordenación del territorio en Europa. Sevilla: Universidad de Sevilla - Junta de Andalucía.

Holling, C. S. (1973). Resilience and stability of ecological systems. Annual Review of Ecology and Systematics, 4, $1-23$.

Hucke-Gaete, R., Ruiz, J. y Álvarez, R. (2010). Descripción de la ecorregión Chiloense. En Hucke-Gaete, R., Lo Moro, P. y Ruiz, J. (Eds.). Conservando el mar de Chiloé, Palena y Guaitecas (pp. 26-62). Puerto Montt: Comisión Nacional del Medio Ambiente.

Ishizaka, A. y Labib, A. (2009). Analytic hierarchy process and expert choice: benefits and limitations. OR Insight, $22,201-220$

Janssen, M. A., Bodin, Ö., Anderies, J. M., Elmqvist, T., Ernstson, H., Mcallister, R. R. J, Olsson, P. y Ryan, P. (2006). A network perspective on the resilience of socialecological systems. Ecology and Society, 11, 15.
Jentoft, S. y Eide, A. (Eds.) (2011). Poverty mosaics: realities and prospects in small-scale fisheries. Dordrecht: Springer Netherland

Kallis, G. y Norgaard, R. (2010). Coevolutionary ecological economics. Ecological Economic, 69, 690-699.

Kelleher, K. (2005). Discards in the world's marine fisheries. An update. Roma: FAO Fisheries Technical Paper.

Kollmann, M. (2005). Una revisión de los conceptos de “territorios equilibrados" y "región”. Procesos de construcción y descontrucción. Theomai, 11, 1-12.

Lamson, C. (1986). Planning for resilient coastal communities: lesson from ecological systems theory. Coastal Zone Management Journal, 13, 265-279.

Lara, C., Miranda, M., Montecino, V. e Iriarte, J. L. (2010). Chlorophyll-a MODIS mesoscale variability in the Inner Sea of Chiloé, Patagonia, Chile (41-43ㅇ): Patches and Gradients? Revista de Biología Marina y Oceanografía, 45, 217-215.

Laterra, P., Orue, M. E. y Booman, G. C. (2012). Spatial complexity and ecosystem services in rural landscapes. Agriculture, Ecosystems \& Environment, 154, 56-67.

Liu, D. y Hao, S. (2017). Ecosystem health assessment at county-scale using the pressure-state-response framework on the Loess Plateau, China. International Journal of Environmental Research and Public Health, 14, 2.

Marín, A. y Berkes, F. (2010). Network approach for understanding small-scale fisheries governance: The case of the Chilean coastal co-management system. Marine Policy, 34, 851-858.

Montero, C. (2004). Formación y desarrollo de un cluster globalizado: el caso de la industria del salmón en Chile. Santiago: Publicaciones CEPAL.

Moreno-Jiménez, J. M., Aguarón, J., Cano, F. y Escobar, M. T. (1998). Validez, robustez y estabilidad en decisión multicriterio. Análisis de sensibilidad en el proceso analítico jerárquico. Revista de la Real Academia de Ciencias Exactas, Fisicas y Naturales, 92, 387-397.

Munda, G. (2004). Métodos y procesos multicriterio para la evaluación social de las políticas públicas. Revista Iberoamericana de Economía Ecológica, 1, 31-45. 
National Research Council (2002). The drama of the commons. Committee on the Human Dimensions of Global Change. Division of behavioural and social sciences and education. Washington: National Academy Press.

Olsson, P. (2003). Building capacity for resilience in socialecological systems. Estocolmo: Stockholm University.

Ostrom, E. (2000). Crowding out citizenship. Scandinavian Political Studies, 23, 3-16.

Papadimitriou, F. (2012a). Modelling landscape complexity for land use management in Rio de Janeiro, Brazil. Land Use Policy, 29, 855-861.

Papadimitriou, F. (2012b). The algorithmic complexity of landscapes. Landscape Research, 37, 591-611.

Pauly, D. (2005). Global trends in world fisheries: impacts on marine ecosystems and food security. Philosophical Transactions of the Royal Society: Biological Sciences, 360, $5-12$.

Pauly, D. (2006). Major trends in small-scale marine fisheries, with emphasis on developing countries, and some implications for the social sciences. Maritime Studies, 4, $7-22$.

Pauly, D., Alder, J., Bennett, E., Christensen, V., Tyedmers, P. y Watson, R. (2006). The Future for fisheries. Science, 302, 1359-1361.

Perry, R. I., Ommer, R. E., Barange, M. y Werner, F. (2010). The challenge of adapting marine social-ecological systems to the additional stress of climate change. Current Opinion in Environmental Sustainability, 2, 356-363.

Plummer, R. (2009). The adaptive co-management process: an initial synthesis of representative models and influential variables. Ecology and Society, 14, 24.

Prescott-Allen, R. (2001). The wellbeing of Nations: a country-by-country index of quality of live and of environment. Washington, DC: Island Press.

Reed, M. S., Stringer, L. C., Dougill, A. J., Perkins, J. S., Atlhopheng, J. R., Mulale, K. y Favretto, N. (2015). Reorienting land degradation towards sustainable land management: Linking sustainable livelihoods with ecosystem services in rangeland systems. Journal of Environmental Management, 151, 472-485.
Santos, M. (1990). Por una Geografía Nueva. Madrid: Espasa-Calpe.

Santos, M. (2000). La naturaleza del espacio: técnica y tiempo, razón y emoción. Barcelona: Ariel Geografía.

Suárez de Vivero, J. L., Rodríguez-Mateos, J. C. y Del Corral, D. F. (2008). La gobernanza en la pesca: De lo ecológico a lo ético, de lo local a lo global. Scripta Nova, $12,256-280$.

Tainter, J. A. (1995). Prehistoric societies as evolving complex systems. En Tainter, J. y Tainter, B. B. (Eds.). Evolving complexity and environmental risk in prehistoric southwest (pp. 1-24). Washington: SFI \& Addison-Wesley Logman.

Ther-Ríos, F. (2006). Complejidad territorial y sustentabilidad: notas para una epistemología de los estudios territoriales. Horizontes Antropológicos, 25, 105-115.

Ther-Ríos, F. (2008). Prácticas cotidianas e imaginarios en sociedades litorales. El sector de Cucao, isla grande de Chiloé. Chungara. Revista de Antropología Chilena, 40, 67-80.

Ther-Ríos, F. (2011). Configuraciones del tiempo en el mar interior de Chiloé y su relación con la apropiación de los territorios marítimos. Desenvolvimento e Meio Ambiente, 23, 67-80.

Urquieta, M. A., Mariñez, C. y Jorquera, C. (2017). Territorio como medium: Discusión sobre rendimientos analíticos para las observaciones de la complejidad socioespacial. Revista Mad. Revista del Magister en Análisis Sistémico Aplicado a la Sociedad, 37, 143-166.

Van Der Leeuw, S. (2000). Land degradation as a socionatural process. En McIntosh, R. J., Tainter, J. A. y McIntosh, S. K. (Eds.). The Way the Wind Blows: Climate, History, and Human Action (pp 357-383). New York: Columbia University Press.

Vayda, A. P. y McCay, B. J. (1975). New directions in the ecology and ecological anthropology. Annual Review of Anthropology, 4, 293-306.

WCED (1987). Our Common Future. Report of the World Commission on Environment and Development. Oxford: Oxford University Press. 
Worm, B., Barbier, E. B., Beaumont, N., Duffy, J. E., Folke, C., Halpern, B. S., Jackson, J. B. C., Lotze, H. K., Micheli, F., Palumbi, S. R., Sala, E., Selkoe, K. A., Stachovicz, J. J. y Watson, R. (2006). Impacts of biodiversity loss on ocean ecosystem services. Science, 314, 787-790.

Young, O., Berkhout, F., Gallopin, G., Janssen, M., Ostrom, E. y Van Der Leeuw, S. (2006). The globalization of socio-ecological systems: An agenda for scientific research. Global Environmental Change, 16, 304-316.
Zeller, D., Booth, S. y Pauly, D. (2005). Underestimating Small-Scale Fisheries: Contributions to GDP. Fisheries Centre Working Paper FAO. Vancouver, Canadá: University of British Columbia.

Zimmerer, K. S. (1994). Human geography and the 'new ecology': the prospect and promise of integration. Annals of the Association of American Geographers, 84, 108-125.

Zolli, A. y Healy, A. M. (2012). Resiliencia: por qué las cosas vuelven a su lugar. Bogotá: Norma. 